\title{
Characterizations of Flexible Wearable Antenna based on Rubber Substrate
}

\author{
Saadat Hanif Dar, Jameel Ahmed \\ Department of Electrical Engineering \\ RIPHAH International University \\ Islamabad, Pakistan
}

\begin{abstract}
Modern ages have observed excessive attention from both scientific and academic communities in the field of flexible electronic based systems. Most progressive flexible electronic systems require incorporating the flexible rubber substrate antenna operating in explicit bands to offer wireless connectivity which is extremely required by today's network concerned society. This paper characterizes flexible antenna performance under the environments developed by natural rubber as the substrate. Flexible antenna grounded on rubber substrate was simulated using CST microwave studio with diverse permittivity and loss tangent. In our work, prototype antennas were built using natural rubber with different carbon filler substances. This paper reveals advanced flexible substrate effects on antenna quality factor $(Q)$ and its consequences on bandwidth and gain. Such antennas under bending washing environment were also found to perform better than existing designs, showing less change in their gain, frequency shift and impedance mismatch.
\end{abstract}

Keywords-wearable antenna; antenna characterization; antennas

\section{INTRODUCTION}

Wearable and elastic wireless communication systems are gaining exceptional popularity due to their thoughtful prospective in daily life particularly in health care monitoring systems.Economic co-operation and Development (OCED) and BRIICS (Brazil, Russia, Indonesia, India and South Africa) are spending $6 \%$ of their GDP to address long term health care issues and are anticipated to escalate $14 \%$ in the next 50 years [1].Chronic disease identification and treatment through Remote patient monitoring (RPM) systems are the primary reasons of this increase.

Modern Flexible Electronics such as flexible mobile phones, electronics books and roll able keyboards are fitted out with an antenna to provide wireless connectivity. The competence of such systems depends on the characteristics of the integrated antenna near human body.Various design parameters of flexible wearable antenna is an important area that required to be evaluated as the different steps involved in its designing are relatively different from the rigid substrate oriented antennas [2].The broad design procedure of flexible and wearable antennas is depicted in figure. 1

\author{
Muhammad Raees \\ Department of Software Engineering \\ Mirpur University of Science and Technology (MUST) \\ Mirpur AJK, Pakistan
}

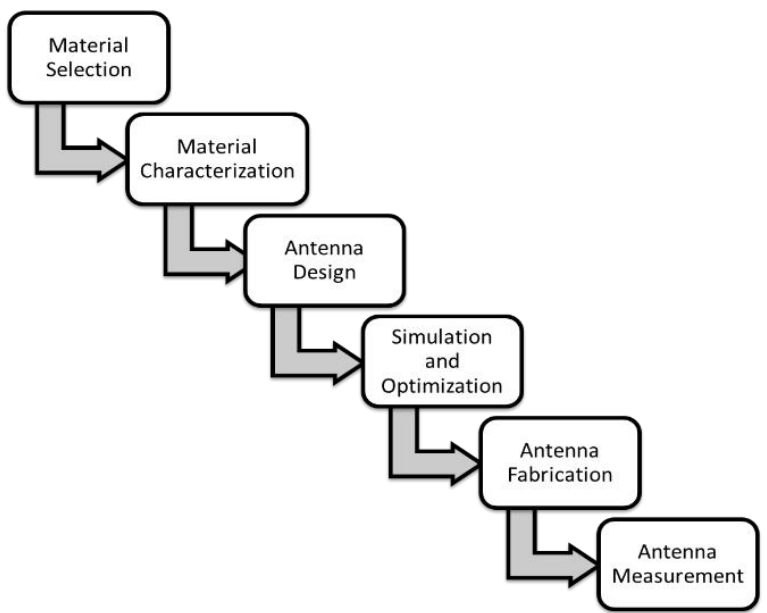

Fig. 1. Design procedure of flexible wearable antenna (Reproduced from [2])

The process starts with the selection and dielectric characteristics (electric conductivity, loss tangent, relative permittivity) of the conductive and substrate material.In the next step antenna geometry, ground plane, radiating element and feeding structure is defined. Finally, the critical parameters (durability, humidity, bending effects) are performed near human body.

Bendable wearable materials like conductive fibers, liquid metallic blends, and polymer in paper [3-6] are widely used in the existing flexible electronic devices. However, additional requirements are enforced in wearable applications. Therefore, these wearable applications require small size, light weight and low profile antennas, which must present stable electrical properties, low power consumption, reasonable impedance match and desirable radiation. Natural rubber is an attractive alternate flexible material which is biocompatible and offers high conductivity, low lost, ease to manufacture and the most important it is water/weather resistant and environment friendly.

Natural rubber is a very common material, however, its application in flexible electronics is very limited, and to our knowledge, flexible wearable antenna presented in [7] is the 
first demonstration made so for to develop flexible antenna with rubber material. Rubber based substrates are insulators in nature since the atoms in rubber chain are covalent bonded. Conductive fillers such as carbon fibers or metal oxides are introduced to form the conductive paths in rubber [6,7].Carbon packings imparts a significant effect on the microwave characterization of natural rubber and hence increase in Tan $\delta$ and electrical conductivity, as reported in [8].

In this paper, Natural rubber is characterized to design flexible wearable antenna for on-body communications. We conclude our study by reporting characterization of actual antenna prototype, including details of fabrication processes, dielectric properties of the substrate and the consequence of filler contents on antenna quality factor (Q).Further to this, different challenging factors for flexible antennas like bending, wrinkling, Wash ability and environmental factors (humidity and thermal effects) that effects on antenna efficiency and gain in addition to the return loss, radiation pattern are also investigated.

\section{SELECTION CRITERIA FOR SUBSTRATE}

Body area networks (BAN's) require effective wireless connectivity to integrate wearable textile antennas in flexible electronic systems. Rubber substrate is a flexible material and its thickness might change with low pressure.

\section{A. Permittivity}

The permittivity, $\varepsilon$ is a multifaceted parameter and its relative permittivity is formulated as

$$
\varepsilon_{\mathrm{r}}: \varepsilon=\varepsilon_{0} \quad \varepsilon_{\mathrm{r}}=\varepsilon_{0}\left(\varepsilon^{\prime}{ }_{\mathrm{r}}-\mathrm{j}_{\varepsilon}{ }^{\prime \prime}{ }_{\mathrm{r}}\right)
$$

whereas $\varepsilon_{0}$ represents permittivity of vacuum, which is $8.854 \times 10-12 \mathrm{~F} / \mathrm{m}$ [9]. Dielectric constant of the various flexible substrate lies in the range of $2.2<\varepsilon_{\mathrm{r}}<12$ [10].

The lower dielectric constant declines the surface wave losses which are tied to guided wave broadcast within substrate. Therefore; lower dielectric constant raises the impedance bandwidth of the antenna with adequate competence and high gain [11].

Fig. 2 demonstrates the deviation of $\varepsilon$ with frequency for diverse filler contents in rubber substrate. Significant change in $\varepsilon_{\mathrm{r}}^{\prime}$ is observed by changing filler contents from $0 \%$ to $60 \%$. Relative permittivity is enhanced when the filler contents are increased.EM signals can move through the material easily and henceforward this will result to lower the permittivity. As obvious from the figure, by increasing carbon filler in natural rubber structure of the substantial material becomes more dense and porous.

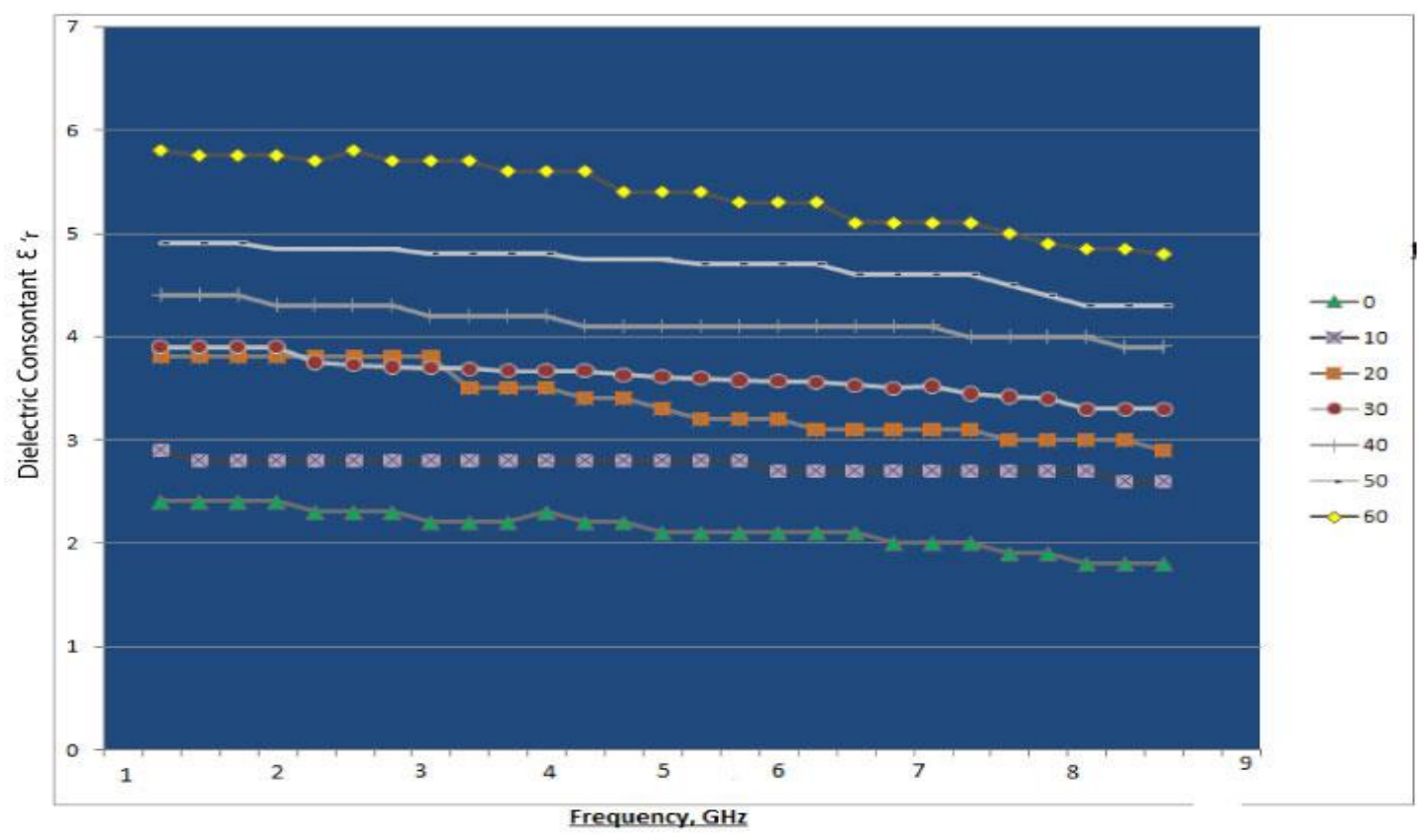

Fig. 2. Relative Permittivity \&r relation with carbon filler components

\section{B. Loss Tangent}

Loss Tangent $(\tan \delta)$ is also identified as dissipation factor. It describes the amount of power turned into heat in the substantial material. Loss tangent in the following relation is defined as the ratio of the imaginary part to real part of the relative permittivity.

$$
\operatorname{Tan} \delta=\varepsilon^{\prime \prime} / \varepsilon_{r}^{\prime}
$$

The higher values of loss tangent results in additional losses in the dielectric substrate and higher losses outcomes in reduced radiation efficiency. The higher losses in tangent values lead to the more losses in dielectric substrate [12] and as a result antenna efficiency reduces.

Figure 3 plots deviation of relative permittivity against the frequency.As perceived from the figure, by increasing the rubber filler contents significant increase in $\tan \delta$ is observed. 
The statement that with the addition and increment of rubber filler contents material gets loss and this leads to an increase in electrical conductivity, reported in [12].Since the carbon contents impacts electrical conductivity and as a result the dielectric properties of the rubber and hence effects on bandwidth, return loss and quality factor of antenna.

\section{Thickness of the Dielectric Fabrics}

The bandwidth and competence of a natural rubber based flexible antenna is principally decided by the substantial substrate dielectric constant and its width. The width $h$ of substrate normally lies in the range of $0.003 \lambda \leqslant \mathrm{h} \leqslant 0.005 \lambda$ whereas $\lambda$ represents its wavelength. For a fixed comparative permittivity, the substrate thickness may be selected to maximize the bandwidth of the flexible antenna. Though, this value may not improve the antenna efficiency.

The effect of the thickness on the bandwidth (BW) of the antenna could be described by Equation (2), where $\mathrm{Q}$ is the antenna quality factor.

$$
B W \sim 1 / Q
$$

The quality factor $(\mathrm{Q})$ is prejudiced by the space wave losses, surface wave and dielectric losses. The selection of the thickness of the dielectric substantial material is a negotiation between competence and bandwidth of the antenna.

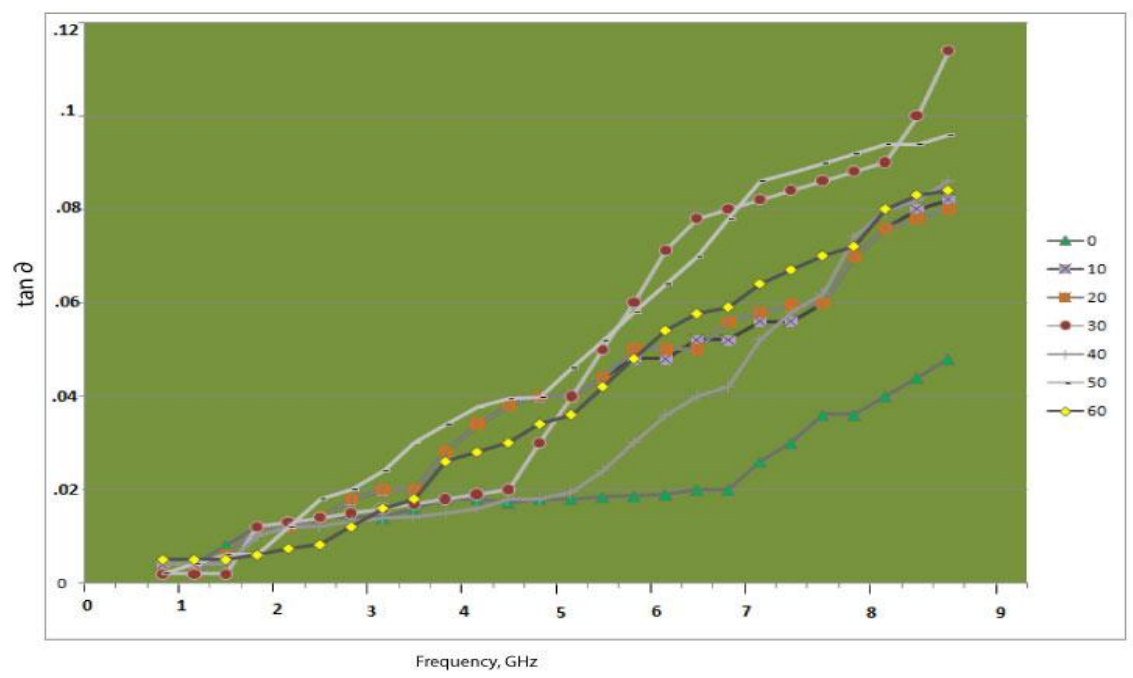

Fig. 3. Tangent loss (Tan $\delta$ ) relation with frequency on different carbon compositions

\section{ANTENNA DESIGN CRITERIA}

Micro strip patch antenna design is used to present natural rubber based flexible wearable antenna.Several types of antennas like micro strip patch antennas were designed by using rubber in CST Microwave Studio Software. Wire antennas in stand-alone approach experiences shift in frequency due to variation of wavelength, which depends on distance from human body. However, rubber based flexible antenna experiences less effects in on-body communications. Its major effect is related with the location of antenna on the body and type of antenna being used. Natural rubber based patch antenna due to full ground plan reduces back radiations while placing near human body [13]. It is exciting that Flexible antennas designed with natural rubber are suitable candidate for wearable antennas in Body Area Networks (BAN's).

The geometry of Flexible Antenna shown in figure. 4 aims to investigate wearable patch antenna with different height of substrate.Each antenna had the same path size however; its relative permittivity is varying. The antenna feature a simple copper patch which act a radiator, fed by $50 \Omega$ inset feed line. Antenna dimensions are calculated by using the transmission line model based on substantial substrate permittivity and loss tangent of 3.2 and 0.01 .

To design a patch rubber based antenna approximate value of dielectric is taken into account. The dielectric constant value of the natural rubber substare may be calculated by simply measuring the resonant frequency of small area radiator. Rubber patched antennas are designed by calculating its various dimensions. The patch width (w) imparts a slight effect on the resonant frequency (fr), and it is designed by using the following formula [14].

$$
w=\frac{c}{2 f r} \sqrt{\frac{2}{(\varepsilon r+1)}}
$$

Width (W) and length (L) of the patch are the major ingredients which characterizes the antenna design. Width of the radiating patch imparts slight effect on the radiation pattern shapes, but has major effect on the input impedance and operating bands.

Radiation power of the antenna increases as the width of the radiator in increased. As a result, it expands bandwidth and increases efficiency. It is supposed that ratio of width to length lies in the range of $1<\mathrm{W} / \mathrm{L}<2$.

Width (W) and length (L) parametric values satisfy the above conditions in the following equations:

$$
L=\frac{C}{2 f r \sqrt{\text { घre }}}-2 \Delta \mathrm{L}
$$

Where, sre is the effective dielectric constant and is calculated by using the following relation 
$\varepsilon r e=(\varepsilon r+1) / 2+(\varepsilon r-1) / 2(1+12 \mathrm{~h} / \mathrm{w}) 1 / 2$

(5)

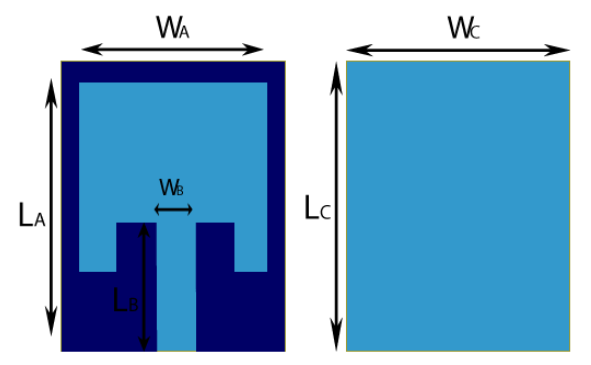

Fig. 4. Antenna Design (a) front view and (b) back view

\section{RESULTS AND DISCUSSIONS}

To Study the effect of rubber substrate, flexible wearable antenna simulations were conducted by using commercially existing CST studio to disclose several dielectric properties, and further to calculate their impacts on the antenna performance in terms of quality factor $(\mathrm{Q})$.

Figure 5 and Figure 6 shows the simulated and measured rejoinders of the antenna correspondingly. The following assumptions can be made from the found results.

1) The band-width increases with the addition of carbon contents in the rubber substrate.As the carbon contents increases, Tan $\delta$ increases and the rubber substrate gets loss and hence the $\mathrm{Q}$ decreases.

2) The return loss degraded with the accumulation in rubber contents. As, the rubber contents increases, the substrate resistivity and permittivity changes, leading to variation in impedance match.

3) The resonant factor is also affected by carbon contents. The shift in frequency is enlightened by the act as the permittivity changes, the wavelength variations as well, giving increase to frequency.

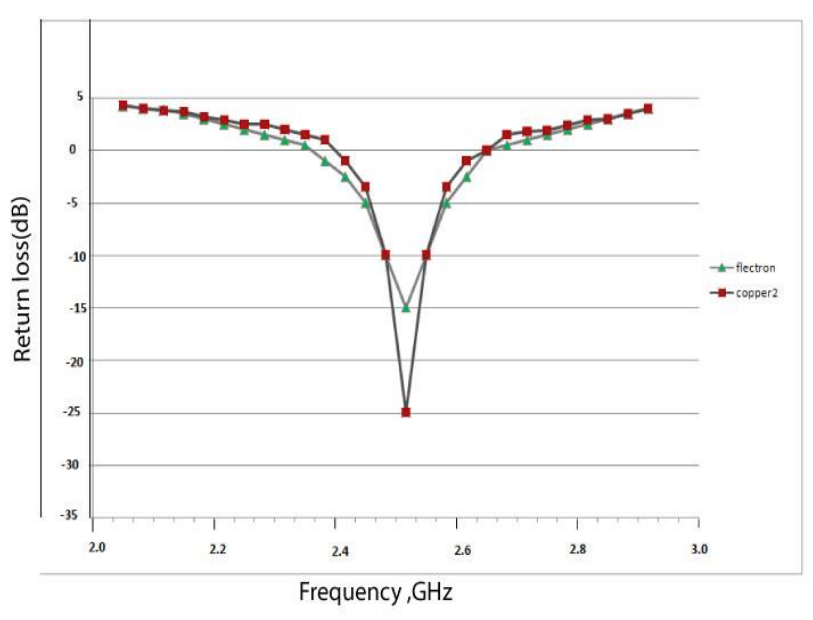

Fig. 5. Simulated return loss of the proposed antenna with diverse contents of rubber

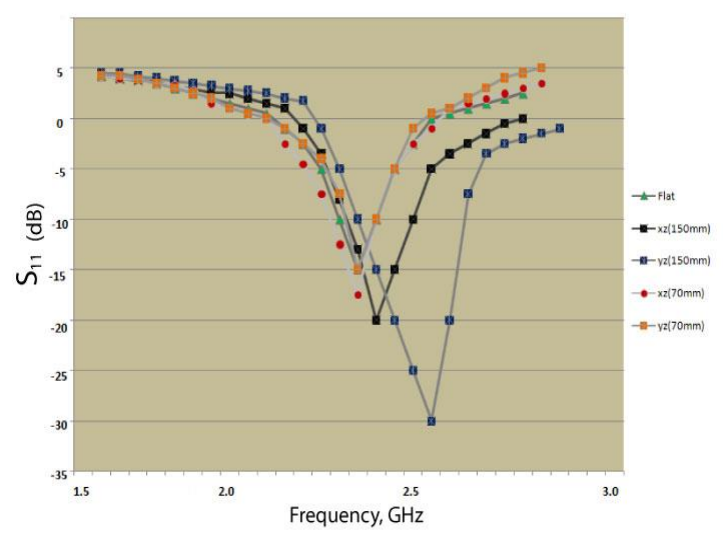

Fig. 6. Simulated return loss of optimized antenna

The prototype antenna is analyzed based on return loss and gain. The bandwidth for the antenna is increased as Tand of the rubber substrate were increased. Characteristically, the total Q is given by the following equation. 


$$
1 / Q=1 / Q_{\text {rad }}+1 / Q_{d}+1 / Q_{c}+1 / Q_{s w}
$$

Where, $\mathrm{Q}_{\mathrm{rad}}, \mathrm{Q}_{\mathrm{c}}, \mathrm{Q}_{\mathrm{d}}$ and $\mathrm{Q}_{\mathrm{sw}}$ are the performance parameters of flexible antenna .The $\mathrm{Q}$ of the dielectric $\left(\mathrm{Q}_{\mathrm{d}}\right)$ is inversely proportional to Tan $\delta$ of dielectric concluded in equation 7 .

$$
Q_{d}=1 / \operatorname{Tan} \delta
$$

Therefore, the rise in rubber contents causes the increase of electrical conductivity of substrate and as a result increases in Tan$\delta$. Finally, the bandwidth is increased because bandwidth (BW) is inversely proportional to Voltage-standing-waveratio(VSWR), shown in equation 8.

$$
B W=(V S W R-1) /(Q \sqrt{ } V S W R)
$$

\section{OPPORTUNITSIC CHALLENGES OF WEARABLE ANTENNA}

\section{A. Bending and crumpling effects}

When wearable flexible antennas are operated on human body, bending, crumpling and sometimes, twisting actions are inevitable.For practical applications, the changes impart adverse effects on the antenna performance.

Detailed technique on the flexible antenna tests reported in [15] is briefed as follows:

1) Robustness and strength tests are executed by repeated trials of the fabrication antenna under bending, crumpling, and twisting to guarantee there are no wrinkles which might reduce the antenna's performance.

2) Resonant frequency and return loss are characterized under twisting conditions since they are inclined to weaken due to impedance discrepancy and capacitive coupling. Bending tests are conducted by confirming the antenna under test on foam cylinders of different radii to parodist diverse bending degrees. The impedance matching declines with a shift in operating frequency as the flexible antenna under goes bending and crumpling circumstances.

3) On the other hand, wrinkling formers are usually engaged to measure the antenna performance under bending and crumpling conditions. The crumpling results in by varying in different directions. The impedance matching declines with a shift in operating frequency as the flexible antenna under goes bending and crumpling circumstances.

The radiation efficiency varies consequently and therefore radiation patterns are distorted. The volume of distortion signal is dependent on the magnitude of bending and crumpling, as reported in [16].

\section{B. Extensive Tests for Flexible Wearable Antenna}

Supplementary measurements are needed when flexible antenna is operated in a specific situation. Here, two extensively conducted tests are introduced:

\section{1) Washing effects (Washability)}

Wearable bendable textile-based antennas are typically uncovered to dust, dirt and excretion, which might compromise their performance. Furthermore, wearable Textile antennas that are merged within clothing are subject to be soaked with water and or wash away. The performance of antenna is obligatory to be consistent after it is washed. To measure the antenna performance after going through wet conditions, its Wash ability test is reported in [17].Apparently the antenna performance in such conditions depends on the conductive and substrate substantial choice.

\section{2) Atmospheric effects (Moisture and Temperature tests)}

Wearable flexible Martials are sensitive to the environmental variables, such as moisture and temperature. As in more or less suitcases wireless systems are required to operate under tough environmental conditions. Performance constancy tests are indispensable.

When an antenna is operated in moist and hot weather atmosphere, the impact of relative humidity and temperature should be examined.

In [18], the effect of moisture and dampness on the reflection coefficient are explored by varying humidity level from $10 \%$ to $90 \%$.Apparently, with increased relative moisture, the permittivity and the loss tangent are both amplified.

\section{CONCLUSION}

This Paper has presented a thorough study on flexible wearable antenna built from a new substantial substrate (rubber).Antenna prototype was presented and its performance near human body is investigated.

The effect of rubber contents on antenna quality factor and bandwidth were pragmatic. With enhanced processing techniques, it should be possible, in theory, to produce rubber with better dielectric properties, and therefore antennas with better performance should be appreciated. It is observed that bandwidth and return loss are improved by adding rubber contents. The antenna efficiency and hence its gain decreased, but this is considered reasonable since natural rubber is quite loss in nature. With improved processing techniques, it should be possible, in theory, to produce rubber with better dielectric properties, and as a result antennas with better performance should be realized.

However, it is pertinent to mention that the antenna performed better when they were subjected to bending and washing conditions than antennas with other substrate. In summary, we have proved the viability of using a new natural material in designing bendable antenna that can tolerate under washing and other environmental conditions like humidity. We have established that antenna performance can be well-ordered by adding rubber contents.

\section{REFERENCES}

[1] de la Maisonneuve, C., Martins, J.O., "Public spending on health and long-term care: a new set of projections,"OECD Economic Policy Papers, no. 6. (2013) http://www.oecd.org/economy/public-spendingon-health-and-long-term-care.htm .

[2] H.Khaleel, Innovation in Wearable and Flexible Antennas:, Ser.Wit Transaction on State-Of-the-art in Science and Engineering, WIT press, 2015.

[3] Liyakath, R. A., A. Takshi, and G. Mumcu, "Multilayer stretchable conductors on polymer substrates for conformal and reconfigurable antennas," IEEE Antennas and Wireless Propag. Lett., Vol. 12, 603606, 2013. 
[4] Hayes, G. J., A. Qusba, M. D. Dickey, and G. Lazzi, "Flexible liquid metal alloy (EGaIn) microstrip patch antenna," IEEE Trans. Antennas Propag., Vol. 60, No. 5, 2151-2156, May 2012.

[5] Hazra, R., C. K. Ghosh, and S. K. Parui, "Effect of different semi conductive substrate materials on a P-shaped wearable antenna," Int. J. of Adv. Res. in Comp. and Comm. Eng. (IJARCCE), Vol. 2, No. 8, 3071-3074, 2013

[6] Xi, J., H. Zhu, and T. T. Ye, "Exploration of printing-friendly RFID antenna designs on paper substrates," IEEE Int. Conf. on RFID, 38-44, April 2011.

[7] Zaiki Awang, Nur A.M.Affendi and Nur M.Razali, "Flexible Antenna Based on Natural Rubber,"Progress In Electromagnetic Research C, Vol.61,75-90,2016.

[8] Olivera,F.A.,N.Alves,J.A.Giacometti,C.J.L Constantino, L.H.C. Mattoso, A.M.O.Balan,and A.E job, "Study of the thermomechanical and electrical properties of conducting composites containing natural rubber and carbon black,"J.Appl.Polym.Sci.,Vol.106,No.2,1001$1006,2007$.

[9] Rais,N.H.M.Soh,P.J.Maliek,F.Ahmad.S.Hashim,N.B.M.Hall,P.S “A review of Wearable Antenna",Antenna \& Propagation Conference, 2009,pp 225-228.

[10] C.A.Blannis, "Antenna Theory :Analysis and Design",3rd ed.,,Wiley,2005,pp.770.
[11] Baker-Jarvis ; Janezic, M.D.; DeGroot, D.C. High-Frequency Dielectric Measurements. IEEE Trans. Instrum. Meas. 2010, 13, 24-31.

[12] B.Gupta,S.Sankarlinngam,S.Dhar , "In proceedings of Mediterranean Microwave Symposium (MMS), Turckey,2010,pp.251-267.

[13] Affendi, N. A.M., N. A. L. Alias, Z. Awang, M. T. Ali, and A. Samsuri, "Microwave non-destructing testing of rubber at X-band," 2013 IEEE Int. RF Microw. Conf., 333-337, Penang, December 2013.

[14] Jaime G.Santas, Akram Alomaniny and Yang Hao, "Textile Antenna for on-body communications: Techniques and properties",EuCAP,2007

[15] Khaleel,H.R.,Al-Rizzo,H.\&Rucker,D.,Compact polyimide based antennas for flexible displays.IEEE journal of Display Technology,8(2),pp.91-97,2012

[16] Bai,Q.\&Langley,R.Crumpling of PIFA textile antenna.IEEE Transaction on Antennas and Propagation,60(1),pp.63-70,2012

[17] Scarpello,M.,Kazani,I.Hertleer,C.,Rogier,H.\&Ginste,D.,Stability and efficiency of screen-printed wearable and washable antennas.IEEE Antennas and wireless Propagation Letters,11,pp.838-841,2012

[18] Hertleer,C.,Van Laere,A.,Rogier,H.\&Van Langenhove,L., Influence of relative humidity on textile antenna performance.Textile Res.J.,80(2),pp.177-183,2010 